\title{
Pseudocritical Behavior and Unbinding of Phospholipid Bilayers
}

Lemmich, Jesper; Mortensen, Kell; Ipsen, John Hjorth; Callisen, Thomas Hønger; Bauer, Rogert; Mouritsen, Ole G.

Published in:

Physical Review Letters

Link to article, DOI:

10.1103/PhysRevLett.75.3958

Publication date:

1995

Document Version

Publisher's PDF, also known as Version of record

Link back to DTU Orbit

Citation $(A P A)$ :

Lemmich, J., Mortensen, K., Ipsen, J. H., Callisen, T. H., Bauer, R., \& Mouritsen, O. G. (1995). Pseudocritical Behavior and Unbinding of Phospholipid Bilayers. Physical Review Letters, 75(21), 3958-3961.

https://doi.org/10.1103/PhysRevLett.75.3958

\section{General rights}

Copyright and moral rights for the publications made accessible in the public portal are retained by the authors and/or other copyright owners and it is a condition of accessing publications that users recognise and abide by the legal requirements associated with these rights.

- Users may download and print one copy of any publication from the public portal for the purpose of private study or research.

- You may not further distribute the material or use it for any profit-making activity or commercial gain

- You may freely distribute the URL identifying the publication in the public portal

If you believe that this document breaches copyright please contact us providing details, and we will remove access to the work immediately and investigate your claim 


\title{
Pseudocritical Behavior and Unbinding of Phospholipid Bilayers
}

\author{
Jesper Lemmich, ${ }^{1}$ Kell Mortensen, ${ }^{2}$ John Hjort Ipsen, ${ }^{1}$ Thomas Hønger, ${ }^{1}$ Rogert Bauer, ${ }^{3}$ and Ole G. Mouritsen ${ }^{1}$ \\ ${ }^{1}$ Department of Physical Chemistry, The Technical University of Denmark, Building 206, DK-2800 Lyngby, Denmark \\ ${ }^{2}$ Department of Solid State Physics, Ris $\phi$ National Laboratory, DK-4000 Roskilde, Denmark \\ ${ }^{3}$ Department of Physics, Royal Veterinary and Agricultural University of Denmark, \\ Thorvaldsensvej 40, DK-1871 Frederiksberg, Denmark \\ (Received 30 March 1995)
}

\begin{abstract}
The temperature dependence of the small-angle neutron scattering from fully hydrated multilamellar phospholipid bilayers near the main phase transition is analyzed by means of a simple geometric model which yields both the lamellar repeat distance as well as the hydrophobic thickness of the bilayer and the interlamellar aqueous spacing. The results for two different lipids show that the lamellar repeat distance displays pseudocritical behavior with a value of the exponent that is in accordance with the theory for critical unbinding transitions of interacting membranes.
\end{abstract}

PACS numbers: 87.64.Bx, 64.60.Fr

Phospholipid bilayers in aqueous solution display thermal phase transitions, one of which is the main phase transition [1,2]. During this transition, which predominantly involves chain melting within the individual bilayers, the average area per molecule increases concomitantly with a decrease in the hydrophobic bilayer thickness (the average lipid acyl-chain length). Results from a variety of experimental and theoretical studies have pointed to the possibility that the main transition in saturated phospholipid bilayers, although of first order, is close to a critical point [3]. Moreover, the bilayers seem to get closer to this critical point as the acyl-chain length is decreased $[4,5]$. The proximity to a critical point has dramatic consequences for the physical properties both of the individual bilayer as well as for the collective properties of a multilamellar array. Specifically at the transition temperature, $T_{m}$, response functions such as specific heat and lateral bilayer compressibility [2] blow up, order parameters vary dramatically [4], and material properties such as bending rigidity get anomalously small [6]. A particular consequence of the lowering of the bending rigidity is an enhancement of the interlamellar entropic repulsion forces [7], and an anomalous swelling behavior arises [8,9].

In the present Letter we present results obtained from analysis of the small-angle neutron scattering (SANS) from multilamellar arrays of bilayers of deuterated phospholipids, specifically DMPC- $d_{54}$ and DPPC- $d_{62}$ [10], in their main transition region. The analysis is carried out in terms of a one-dimensional geometric model of the scattering intensity function, $I(q)$, which identifies different layers (cf. inset in Fig. 2) within each repeat unit of the multilamellar stack: the hydrophobic lipid-bilayer core of thickness $d_{L}$, two polar-headgroup regions each of thickness $d_{H}$, and the interlamellar water layer of thickness $d_{W}$. From a similar type of SANS experiment [8], we have previously determined the total lamellar repeat distance, $d=d_{L}+2 d_{H}+d_{W}$, whose determination does not require a specific model since it can readily be estimated fairly accurately from the positions of the Bragg peaks in the scattering function. The main result from the present analysis is that the lamellar repeat distance, $d$, for DMPC and the total thickness of the hydrophilic aqueous layers, $d_{A}=2 d_{H}+d_{W}$, for both DMPC and DPPC display distinct peaks at $T_{m}$. In contrast, the hydrophobic bilayer thickness, $d_{L}$, varies monotonously with temperature through the transition region, although rather abruptly on the fluid side of $T_{m}$, which is in good quantitative agreement with results from ${ }^{2} \mathrm{H}$ NMR measurements [4]. We refer to the peak behavior in $d$ and $d_{A}$ as anomalous swelling. The anomaly on the fluid side of the transition can for both DMPC and DPPC be rationalized in terms of pseudocritical behavior

$$
d(T)-d_{0} \sim\left(T-T^{*}\right)^{-\psi}, \quad T \rightarrow T_{+}^{*},
$$

with $\psi=1$, where the pseudocritical temperature, $T^{*}$, is found to be very close to the equilibrium transition temperature, $\left(T_{m}-T^{*}\right) / T_{m} \simeq 10^{-2}$. In Eq. (1), $d_{0}$ is the repeat distance in the fluid phase far from the phase transition. We suggest that the observation of the onset to a power-law singularity for the lamellar repeat distance, Eq. (1), which, however, only partially develops because of the intervention of a first-order phase transition, may be understood within the framework of the theory of critical unbinding transitions for interacting membranes $[11,12]$. We believe that our results in terms of Eq. (1) with the exponent value $\psi=1$ represent the first experimental observation of a phenomenon that in a quantitative manner can be directly related to critical unbinding of interacting lipid membranes.

The samples of nonoriented multilamellar bilayers (vesicles) were prepared under excess water conditions by dissolving dry lipid in a $\mathrm{D}_{2} \mathrm{O}$ buffer solution [13]. Two independent preparations were made for each of the two lipids studied. All four samples were investigated by SANS, and the phase transition temperatures were checked by differential-scanning calorimetry. The SANS measurements, which were carried out at the Ris $\varnothing$ SANS 
facility, were performed for two different neutron wavelengths, 2.8 and $9 \AA$, with sample-to-detector distances of 3 and $1 \mathrm{~m}$. This setup leads to scattering vectors in a range $0.0077<q<0.59 \AA^{-1}$, which in contrast to earlier studies [8] is wide enough to permit a detailed analysis in terms of our geometric model. The data were corrected for background, and instrumental smearing was taken into account in the analysis. Three orders of Bragg reflection can be discerned in the isotropic scattering intensity function $I(q)$.

The analysis of the scattering data is based on the assumption that the system is a nonoriented sample (powder) consisting of stacks of fully hydrated bilayers. The different stacks are parts of large multilamellar vesicles with radii of curvature that are large compared to the thickness of the lamellae. Each stack consists of a set of $N$ repeat units of approximately flat bilayers of finite thickness. Within this effectively one-dimensional geometry the powder-averaged intensity can be written as $I(q) \propto q^{-2} I_{1}(q)$, where $I_{1}(q)$ is the (oriented) intensity function measured in a direction normal to the plane of the lamellae. We have analyzed $I_{1}$ [14] in terms of a paracrystalline theory [15] in which the layered structure is considered within a one-dimensional stacking model [16], i.e., the total scattering intensity is treated without decoupling the form and structure factors. Therefore, our approach [14] is conceptually different from that of Nallet, Laversanne, and Roux [17] and Zhang et al. [9,18] who decouple the form and structure factors. These authors describe the form factor by a model with some degree of internal structure, and for the structure factor they use a Hamiltonian approach based on the Caille theory for the scattering from loosely coupled smectic layers [19]. We contend that such a decoupling of the form and structure factors and the interpretation of the scattering in terms of the Caillé theory [9], which builds on a harmonic approximation, are likely to break down in the transition region which is characterized by strongly coupled layers and non-Gaussian fluctuations.

In our theory for $I_{1}$ we invoke a simple geometric model of the layered systems in which each repeat unit is taken to consist of a sandwich of four layers as illustrated in the inset of Fig. 2. The different layers are assumed to fluctuate in thickness according to a Gaussian distribution. Each type of layer is assigned a specific scattering length density whose value has to be determined from the data. Only the relative scattering length density, $b_{r}$, for the headgroup and acyl-chain layers is derived, since, by the Babinet principle, the scattering length density of the solvent can be put to zero. Using this simple model, the scattering intensity, $I(q)$, can be expressed in terms of eight parameters, $b_{r}, N, d_{L}, d_{H}$, and $d_{W}$ together with the Gaussian spreads on the three geometric parameters [14]. In all cases, the fit of this expression for $I(q)$ to the experimental data is found to be very good.
Since the scattering lengths for deuterium, carbon, and oxygen are comparable in size and considerably larger than that for hydrogen, we have included the acyl-oxy group together with the acyl chains in our definition of the bilayer hydrophobic part. Considering that only small amounts of water penetrate deeper than the acyloxy group this leaves the remaining part of the lipid molecule as a hydrophilic moiety with a substantial degree of hydration. Since the degree of hydration varies with temperature, it is conceptually difficult to separate interlamellar water from headgroup material. We have therefore found it desirable to focus on the total thickness of the hydrophilic layers within the repeat unit, i.e., $d_{A}=$ $2 d_{H}+d_{W}$, although the model does provide information on $d_{H}$ and $d_{W}$ separately [14].

In Fig. 1(a) the results for the lamellar repeat distance, $d(T)$, as a function of temperature are shown for the two different lipids. This data set confirms our previous finding, based on a simple Lorentz fit to the Bragg peaks [8], that $d$ in the case of DMPC displays a distinct peak anomaly at the transition. In the case of DPPC, no peak anomaly can be discerned in this data set. It is a common characteristic for both lipids that, whereas $d$ varies slowly

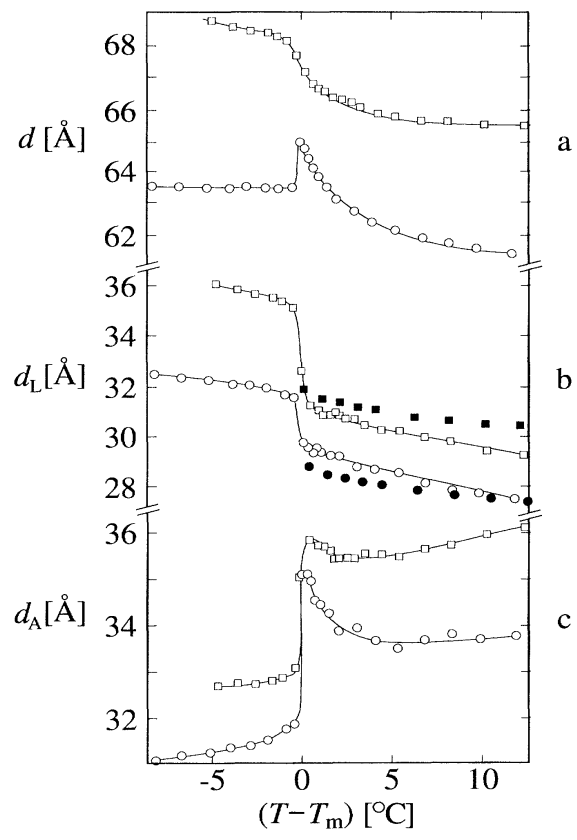

FIG. 1. Lamellar repeat distance, $d$ (a), hydrophobic lipid bilayer thickness, $d_{L}(\mathrm{~b})$, and hydrophilic layer thickness, $d_{A}=$ $d_{W}+2 d_{H}$ (c), as functions of reduced temperature, $T-T_{m}$, for multilamellar bilayers of DMPC- $d_{54}(\circ)$ and DPPC- $d_{62}(\square)$. $T_{m}$ is the main phase transition temperature. In (b) results are shown as obtained from both SANS measurements (open symbols) and ${ }^{2} \mathrm{H}$ NMR measurements (solid symbols) in the fluid phase [4]. Since we have included the acyl-oxy group in the definition of $d_{L}$, whereas in the NMR measurements this part is not included, we have added $2 \times 3 \AA$ to the NMR results, corresponding to $3 \AA$ per acyl-oxy group. 
only with temperature below $T_{m}$, a strong decrease is observed above $T_{m}$. Our data for $d$ are consistent with those reported by Zhang et al. using x-ray scattering [9]. The differences in the actual values of $d$ in the present data set and that previously published [8] using SANS are attributed to differences in the actual samples and details of their preparation.

The results for the hydrophobic bilayer thickness, $d_{L}$, presented in Fig. 1(b) show that for both lipid species the hydrophobic thickness decays monotonously with temperature, with pronounced discontinuities at the respective transition temperature. The discontinuity is larger for the longer lipid, $\Delta d_{L}\left(T=T_{m}\right) \simeq 2 \AA$ for DMPC and $4 \AA$ for DPPC. The variation with temperature is most pronounced above the transition.

The data for the thickness of the hydrophilic aqueous layer, $d_{A}$, shown in Fig. 1(c) demonstrate that the source for the anomalous peak in the repeat distance for DMPC seen in Fig. 1(a) is a blowup in the total thickness of the hydrophilic layers. The data for $d_{A}$ in the case of DPPC also display a weak peak. However, this is not strong enough to manifest itself in the repeat distance because of the large discontinuity in $d_{L}$ at $T_{m}$ for DPPC.

The peak in $d_{A}$ observed for DMPC at $T_{m}$ justifies the use of the term "anomalous swelling" to describe the peak phenomenon observed in the lamellar repeat distance [8]. In this sense our use of the term is different from that proposed by Zhang et al. [9] who use anomalous swelling to describe the increase in the bilayer thickness as the temperature is reduced towards $T_{m}$. The results in Fig. 1(c) demonstrate that the total hydrophilic layer gets anomalously thick at the phase transition, part of which is due to an increased level of hydration of the polar headgroups. The anomalous swelling behavior is less pronounced for DPPC than for DMPC because DMPC is closer to the critical point [5].

The rapid increase of the repeat distance as the temperature is lowered towards $T_{m}$, cf. Fig. 1(a), is reminiscent of critical-point behavior [9] (pseudocritical behavior). Since the phase transition is of first order [3], the apparent singularity occurs at a temperature, $T^{*}$ (the spinodal point), below $T_{m}$. In Fig. 2 we present the results of an analysis of the repeat distance data in Fig. 1(a) according to the power law in Eq. (1) with $\psi=1$. It is seen that the entire data set for $T>T_{m}$ for both DMPC and DPPC is well described by Eq. (1) with the value of the exponent being $\psi=1$. The data are not sufficiently accurate to sustain a full fit with $\psi$ as a variable. A similar analysis of the swelling data for $d_{A}$ in Fig. 1(c) leads to substantial scatter in the fit which, however, is consistent with Eq. (1) and $\psi=1$.

The value of the exponent, $\psi=1$, in Eq. (1) is consistent with the prediction from a functional renormalizationgroup (RG) treatment of critical unbinding of interacting membranes [11]. Within this theory it was shown that interacting membranes have a single fixed-point potential associated with critical unbinding and there is only one relevant scaling field (of dimension one) associated with this fixed point. A consequence of this general prediction is, for realistic intermembrane potentials [12], that $d \sim\left(y-y_{c}\right)^{-\psi}$, with $\psi=1$. In this expression, the field $y$ is either the Hamaker coefficient or the range of the short-ranged repulsive force, and $y_{c}$ is the corresponding critical field value for which direct potential is mapped onto the same fixed-point potential under the RG transformation. In general, this type of singular behavior is expected for variation of any thermal field that brings the membrane into the critical unbinding transition. For phospholipid bilayers close to the main transition, and therefore close to the pseudocritical point, it is anticipated that the hydration forces, the van der Waals interactions, and in particular the membrane bending rigidity [6], that gives rise to an enhancement of the repulsive undulation force [8], are all changing smoothly with temperature, which is the tunable thermal field in the present type of experiment. Therefore, the RG prediction of critical unbinding behavior induced by changes in the field $y$ immediately carries over to the type of singular behavior in terms of varying $T$ as described by Eq. (1) with $\psi=1$ [12]. Since in the present case, cf. Fig. 1, a first-order transition intervenes before the singular point is reached, the unbinding transition does not fully develop. Still, the characteristic fluctuations of the unbinding transition manifest themselves as a pseudocritical (pretransitional) phenomenon over a remarkable wide range of temperatures in the fluid phase.

From the plots in Fig. 2 the relative distance, $t=$ $\left(T_{m}-T^{*}\right) / T_{m}$, of the pseudocritical point from the equilibrium transition temperature can be determined to be 0.010 and 0.008 for DMPC and DPPC, respectively. Based on the present experimental data, we are unable to assess if the two values quoted are actually different, and

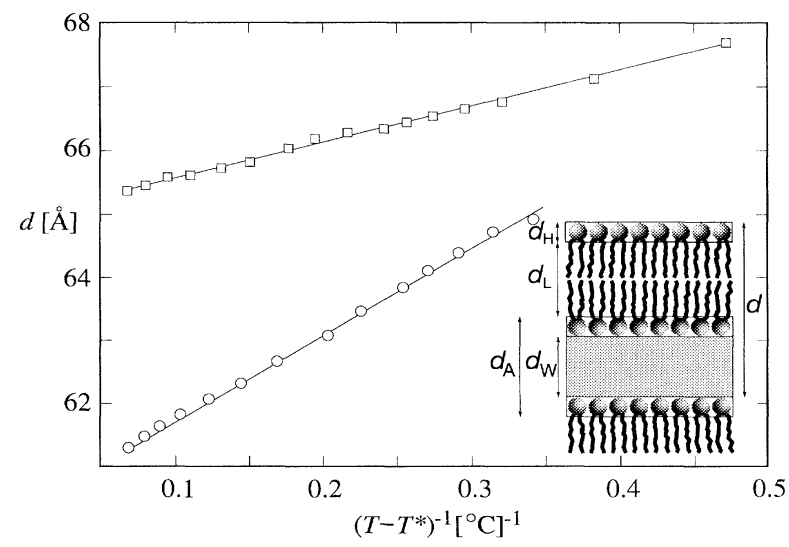

FIG. 2. Plot of reduced lamellar repeat distance, $d(T)$, vs inverse distance, $\left(T-T^{*}\right)^{-1}$, from the pseudocritical temperature, $T^{*}$; cf. Eq. (1). The inset shows a schematic representation of the different layers within a repeat unit of the multilamellar stack. 
we conclude that for both lipid species $t \sim 10^{-2}$. Since the position of the pseudocritical point may depend on the type of physical property one extrapolates beyond the region of thermodynamic stability to determine the locus of the apparent singularity, it is not straightforward to compare with results for $t$ obtained from analysis of other properties [4].

In addition to providing evidence for pseudocritical behavior and partial unbinding in multilamellar lipid bilayers near their phase transition, the present approach has indicated a route to a direct determination of hydrophobic bilayer thickness, $d_{L}$, without making any assumptions about specific volumes of lipid. Information on bilayer hydrophobic thickness is of importance for biophysical studies of the interaction between integral membrane proteins and biological membranes [20]. It has been suggested [21,22] that $d_{L}$ can be estimated from ${ }^{2} \mathrm{H}$ NMR spectroscopy in the fluid phase (where the orientational order monitored by the NMR segmental $C-D$ order parameter, $S_{C D}$, arises from conformational and rotational motions of axial symmetry around the bilayer normal) by a simple linear relationship, $d_{L}=a S_{C D}+b$, where $a$ and $b$ are geometric constants. As clearly pointed out by Nagle [23] this relationship is at best an approximation. The results from the present SANS measurements put us in a position to evaluate the accuracy of the relationship using the NMR results for DMPC and DPPC by Morrow, Whitehead, and Lu [4]. The comparison between the SANS and NMR data for $d_{L}$ in the fluid phase [24] is made in Fig. 1(b), which shows that the two different types of measurements agree resonably well within $0.5-1 \AA$ for both lipids suggesting that the above linear relationship between $S_{C D}$ and $d_{L}$ can conveniently, although with some caution, be used to provide a rough estimate of hydrophobic bilayer thicknesses.

This work was supported by the Danish Natural Science Research Council and the Danish Technical Research Council. O.G. M. is an Associate Fellow of the Canadian Institute for Advanced Research. Mike Morrow is thanked for providing us with the NMR data used to produce Fig. 1(b) and John F. Nagle for providing us with a preprint of Ref. [9] prior to publication.

[1] G. Cevc and D. Marsh, Phospholipid Bilayers: Physical Principles and Models (Wiley, New York, 1987).
[2] M. Bloom, E. Evans, and O.G. Mouritsen, Q. Rev. Biophys. 24, 293 (1991).

[3] O. G. Mouritsen, Chem. Phys. Lipids 57, 178 (1991).

[4] M. R. Morrow, J. P. Whitehead, and D. Lu, Biophys. J. 63, 18 (1992).

[5] J. H. Ipsen, K. Jørgensen, and O. G. Mouritsen, Biophys. J. 58, 1099 (1990).

[6] L. Fernandez-Puente, I. Bivas, M. D. Mitov, and P. Méléard, Europhys. Lett. 28, 181 (1994).

[7] W. Helfrich, Z. Naturforsch. 33A, 305 (1978).

[8] T. Hønger, K. Mortensen, J.H. Ipsen, J. Lemmich, R. Bauer, and O.G. Mouritsen, Phys. Rev. Lett. 72, 3911 (1994).

[9] R. Zhang, W. Sun, S. Tristram-Nagle, L. Headrick, R. M. Suter, and J. F. Nagle, Phys. Rev. Lett. 74, 2832 (1995).

[10] Abbreviations used: DMPC, dimyristoyl phosphatidylcholine; DPPC, dipalmitoyl phosphatidylcholine; with saturated fatty-acid chain lengths corresponding to 14 and 16 carbon atoms, respectively.

[11] R. Lipowsky and S. Leibler, Phys. Rev. Lett. 56, 2541 (1986).

[12] R. Lipowsky, Europhys. Lett. 7, 255 (1988).

[13] It is important in order to observe the equilibrium swelling behavior in the transition region that the lipid dispersion contains sufficient water (excess water) in order to provide full hydration. In the present case we have used samples with about $85 \%$ water.

[14] A detailed description of the model and our analysis will be published elsewhere [J. Lemmich, K. Mortensen, J. H. Ipsen, T. Hønger, R. Bauer, and O. G. Mouritsen (to be published)].

[15] R. Hosemann and S. N. Bagchi, Direct Analysis of Diffraction by Matter (North-Holland, Amsterdam, 1962), p. 408.

[16] R. Brämer, Kolloid Z. Z. Polym. 250, 1034 (1972).

[17] F. Nallet, R. Laversanne, and D. Roux, J. Phys. II (France) 3, 487 (1993).

[18] R. Zhang, R. M. Suter, and J. F. Nagle, Phys. Rev. E 50, 5047 (1994).

[19] A. Caillé, C. R. Acad. Sci. Ser. B 274, 891 (1972).

[20] O. G. Mouritsen and M. Bloom, Annu. Rev. Biophys. Biomol. Struct. 22, 145 (1993).

[21] J. Seelig and A. Seelig, Q. Rev. Biophys. 13, 337 (1980).

[22] J. H. Ipsen, O. G. Mouritsen, and M. Bloom, Biophys. J. 57, 405 (1990).

[23] J. F. Nagle, Biophys. J. 64, 1476 (1993).

[24] The same comparison cannot be made in the lowtemperature phase since in this phase the lipid acyl chains are not oriented normal to the bilayer plane [1] and $d_{L}$ cannot readily be extracted from the NMR data. 


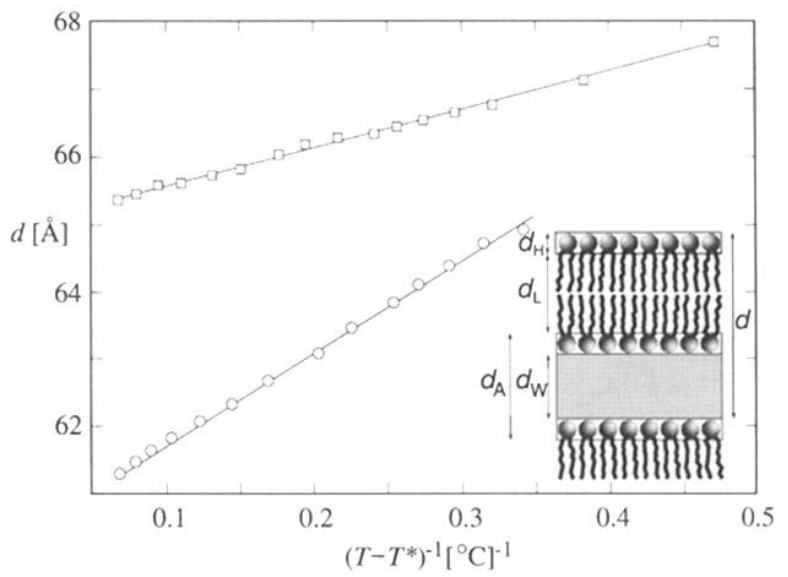

FIG. 2. Plot of reduced lamellar repeat distance, $d(T)$, vs inverse distance, $\left(T-T^{*}\right)^{-1}$, from the pseudocritical temperature, $T^{*}$; cf. Eq. (1). The inset shows a schematic representation of the different layers within a repeat unit of the multilamellar stack. 